\title{
MicroRNA Dysregulation in Liver and Pancreas of CMP-Neu5Ac Hydroxylase Null Mice Disrupts Insulin/PI3K-AKT Signaling
}

\author{
Deug-Nam Kwon, ${ }^{1}$ Byung-Soo Chang, ${ }^{2}$ and Jin-Hoi Kim ${ }^{1}$ \\ ${ }^{1}$ Department of Animal Biotechnology, Konkuk University, Hwayang-dong, Kwangjin-gu, Seoul 143-701, Republic of Korea \\ ${ }^{2}$ Department of Cosmetology, Hanseo University, Seosan, Chungnam 356-706, Republic of Korea \\ Correspondence should be addressed to Jin-Hoi Kim; jhkim541@konkuk.ac.kr
}

Received 16 April 2014; Revised 2 June 2014; Accepted 18 July 2014; Published 28 August 2014

Academic Editor: X. Li

Copyright (c) 2014 Deug-Nam Kwon et al. This is an open access article distributed under the Creative Commons Attribution License, which permits unrestricted use, distribution, and reproduction in any medium, provided the original work is properly cited.

CMP-Neu5Ac hydroxylase (Cmah)-null mice fed with a high-fat diet develop fasting hyperglycemia, glucose intolerance, and pancreatic $\beta$-cell dysfunction and ultimately develop characteristics of type 2 diabetes. The precise metabolic role of the Cmah gene remains poorly understood. This study was designed to investigate the molecular mechanisms through which microRNAs (miRNAs) regulate type 2 diabetes. Expression profiles of miRNAs in Cmah-null mouse livers were compared to those of control mouse livers. Liver miFinder miRNA PCR arrays $(n=6)$ showed that eight miRNA genes were differentially expressed between the two groups. Compared with controls, seven miRNAs were upregulated and one miRNA was downregulated in Cmah-null mice. Specifically, miR-155-5p, miR-425-5p, miR-15a-5p, miR-503-5p, miR-16-5p, miR-29a-3p, and miR-29b-3p were significantly upregulated in the liver and pancreas of Cmah-null mice. These target miRNAs are closely associated with dysregulation of insulin/PI3K-AKT signaling, suggesting that the Cmah-null mice could be a useful model for studying diabetes.

\section{Introduction}

Sialic acids are terminal components of glycoconjugate carbohydrate chains and are involved in cell-cell recognition, ligand-receptor, and cell-pathogen interactions [14]. The most common mammalian sialic acids are $\mathrm{N}$ acetylneuraminic acid (Neu5Ac) and $N$-glycolylneuraminic acid (Neu5Gc). Neu5Gc is generated by hydroxylation of CMP-Neu5Ac to CMP-Neu5Gc, catalyzed by CMP-Neu5Ac hydroxylase (Cmah). An inactivating mutation in the Cmah gene during evolution led to humans losing the common mammalian cell surface molecule $N$-glycolylneuraminic acid (Neu5Gc) $[3,5,6]$. The mutated Cmah gene initiated a series of genetic and biochemical changes in the biology of sialic acids that may contribute to several unique aspects of human biology in health and disease $[7,8]$.

The effects of Cmah deactivation have been observed in Cmah-null mice. These mice have accumulation of Neu5Ac, which is also observed in humans. Similarly Cmah-null mice display human-like phenotypes including diminished acoustic sensitivity [9], altered startle response threshold (relative to normal mice) [9], hearing loss in old age [9], skin healing delay in adulthood [9], and a human-like muscular dystrophy phenotype following combined mutation of the Dmd gene [10]. Immunologically, these mice have enhanced $\mathrm{B}$ cell proliferation and antibody production, consistent with the observation that Neu5Gc is physiologically downregulated during normal B cell activation [11]. A recent study showed that high-fat diet (HFD)-fed Cmah-null mice exhibit fasting hyperglycemia, glucose intolerance, and pancreatic $\beta$ cell dysfunction that ultimately lead to the development of a type 2 diabetes-like syndrome [12]. Therefore, the HFDfed Cmah-null mouse is considered one of the most suitable animal models of the human metabolic syndrome.

MicroRNAs (miRNAs) are small noncoding RNAs with lengths of approximately 22 nucleotides. miRNAs have essential roles in the regulation of diverse biological pathways and play an important role in insulin resistance in various tissues $[13,14]$. For example, in obesity and diabetes, it has been demonstrated that miRNAs such as miR-375, miR-29, miR320 , miR-103, mir-107, and miR-126 play a crucial role in the regulation of glucose and lipid metabolism. This occurs 
through control of pancreatic islet cell function, adipocyte insulin resistance, hepatocyte insulin signaling, and glucose homeostasis [15-19]. miR-33a/b inhibits expression of insulin receptor substrate-2 (IRS-2) in hepatic cells, leading to reduced activation of downstream insulin signaling pathways including AKT and ERK. Antagonism of endogenous miR$33 \mathrm{a} / \mathrm{b}$ upregulates fatty acid oxidation and the response to insulin in hepatocytes, suggesting this is a potential therapeutic target in the metabolic syndrome [20]. However, the precise molecular mechanisms by which miRNAs are involved in insulin resistance in Cmah-null mice remain obscure. The first aim of the present study was to identify changes in miRNA expression in the liver of Cmah-null mice. The second aim was to analyze miRNA expression profiles and to investigate the involvement of the insulin/PI3K-AKT signaling pathway.

\section{Materials and Methods}

2.1. In Vivo Experiments. All lines were maintained on a congenic $\mathrm{C} 57 \mathrm{Bl} / 6 \mathrm{~J}$ background. The mice were allowed to eat and drink ad libitum and were fed standard mouse chow (Cargill Agri Purina, Inc., Seongnam-Si, Korea). Twelve-week-old wild type and $\mathrm{Cmah}$-null male mouse in this study were used. All animal experiments were approved and performed under the guidelines of the Konkuk University Animal Care and Experimentation Community (IACUC approval number: KU12045). Cmah <tm1Ykoz> knockout mice were originally made and kindly provided by RIKEN.

\subsection{MicroRNA Isolation and cDNA Generation. miRNA was} isolated from mouse tissue using the miRNeasy mini kit (Qiagen, Valencia, CA, USA) according to the manufacturer's instruction. Concentration and purity of isolated miRNA were measured using the NanoDrop2000 spectrometer and samples were screened for an A260 : A280 ratio greater than 1.8. Conversion of miRNA to cDNA was carried out using $100 \mathrm{ng}$ of miRNA according to the manufacturer's instructions for the $\mathrm{RT}^{2}$ miRNA First Strand Kit (SA Biosciences, Frederick, MD).

2.3. MicroRNA PCR Array Analysis. Changes in miRNA expression between samples were measured using a Liver miFinder miRNA PCR Array (SA Biosciences, Frederick, MD). Plates contained 84 of the most abundantly expressed and best-characterized miRNAs. The cDNA from individual samples was used as a template for the PCR array using SYBR green, according to the array instructions in an ABI ViiA 7 system (Applied Biosystems, Foster City, CA, USA). Data were analyzed using SABiosciences $\mathrm{RT}^{2}$ Profiler PCR Data Analysis software (available at http://pcrdataanalysis.sabiosciences.com/mirna/arrayanalysis.php) and were considered significant at $>2.0$ fold change. Relative quantitation of each miRNA was determined by normalizing to the small nuclear RNA housekeeping panel (SNORD61, SNORD68, SNORD72, SNORD95, SNORD96A, and RNU6-2) comparing the control wild type (WT) and Cmah-null samples using the $2-\Delta \Delta \mathrm{Ct}$ method.
2.4. Real-Time Reverse Transcription Quantitative Polymerase Chain Reaction (RT-qPCR). The total RNA obtained from tissues (liver and pancreas) was reversely transcribed using the QuantiTect Reverse Transcription Kit (Qiagen, Valencia, USA), according to the manufacturer's instructions. To assess gene expression, real-time reverse transcription polymerase chain reaction was conducted using an ABI ViiA 7 system (Applied Biosystems) with SYBR Green as the doublestranded DNA-specific fluorescent dye (Bio-Rad, Hercules, CA, USA). The mouse Gapdh gene was used as an internal control to normalize the RT-qPCR efficiency and to quantify the expression of mRNAs in WT and Cmah-null tissues. After normalization with Gapdh, the relative expressions of mRNA in Cmah-null tissues were compared with controls. RT-qPCR was performed for each sample in triplicate. The following primers were used: $5^{\prime}$-TCCAAGGGCTACACCAAATC- $3^{\prime}$ and $5^{\prime}$-GTTTTCGAGGGCAGAGACTG-3' for Mapk3; 5' CTACACCCCATGCCTTCACT- $3^{\prime}$ and $5^{\prime}$-GCTGAAGGTGAGGCTGATTC-3' for Raf1; $5^{\prime}$-AGTGCGTGCAGAAGGAGATT- $3^{\prime}$ and $5^{\prime}$-CACAACTTCTCGGCAGTCAA- $3^{\prime}$ for Ccnd2; $5^{\prime}$-CCAGCCTGGCTATTTAGCTG- $3^{\prime}$ and $5^{\prime}$-CCCAACTCAACTCCACCACT- $3^{\prime}$ for IRS1; $5^{\prime}$-ACAACCTATCGTGGCACCTC- $3^{\prime}$ and $5^{\prime}$-GACGGTGGTGGTAGAGGAAA- $3^{\prime}$ for IRS2; $5^{\prime}$-CCCTTCTACAACCAGGACCA-3' and $5^{\prime}$-ATACACATCCTGCCACACGA-3' for AKT1; $5^{\prime}$ GTGAACACCATGCCTCACAC- $3^{\prime}$ and $5^{\prime}$-CACAGTCCAAGCGCTCAATA-3' for FOXO1. Eight miRNA primer sets (Mm_miR-155_1 miScript Primer Assay (MS00001701) for miR-155-5p, Mm_miR-425_2 miScript Primer Assay (MS00012012) for miR-425-5p, Mm_miR-15a_1 miScript Primer Assay (MS00001281) for miR-15a-5p, Mm_miR503_1 (MS00002597) for miR-503-5p, Mm_miR-16_2 (MS00037366) for miR-16-5p, Mm_miR-29a_1 (MS00001372) for miR-29a-3p, Mm_miR-29b_1 (MS00005936) for miR29b-3p, and Mm_miR-880_2 (MS00033103) for miR-880-3p) were obtained from Qiagen (Valencia, CA, USA). To confirm miRNA expression, RT-qPCR was performed on an ABI ViiA 7 system according to standard protocol. Reactions were run in triplicate.

2.5. Western Blot Analysis. Approximately $40 \mathrm{mg}$ of tissue was homogenized using a Tissue-lyser (Qiagen) in ice cold RIPA buffer (with protease inhibitor cocktail) and centrifuged at $13,000 \mathrm{~g}$ for $15 \mathrm{~min}$ at $4^{\circ} \mathrm{C}$ and the pellet discarded. Protein concentration was measured using BCA Protein Assay Kit (Thermo Scientific, Seoul, Korea) and BSA was used to generate a standard curve. Proteins were electrophoresed on $10 \%$ SDS-PAGE gels under nonreducing conditions and were then transferred to PVDF membranes. Blots were incubated with primary antibodies for IRS2 $(1: 1000$, Cell signaling, MA, USA), PI3K (1:1000, Cell signaling, MA, USA), AKT (1:1000, Cell signaling, MA, USA), and p-AKT ( $1: 1000$, Cell signaling, MA, USA), followed by incubation with a secondary goat anti-rabbit IgG antibody conjugated to horseradish peroxidase (HRP) (1:5000, Calbiochem, CA, USA). Bands were visualized using an ECL detection system (Amersham Pharmacia Biotech). An antiactin antibody was used to verify equal protein loading. Band intensity was quantified using ImageJ v1.32. 


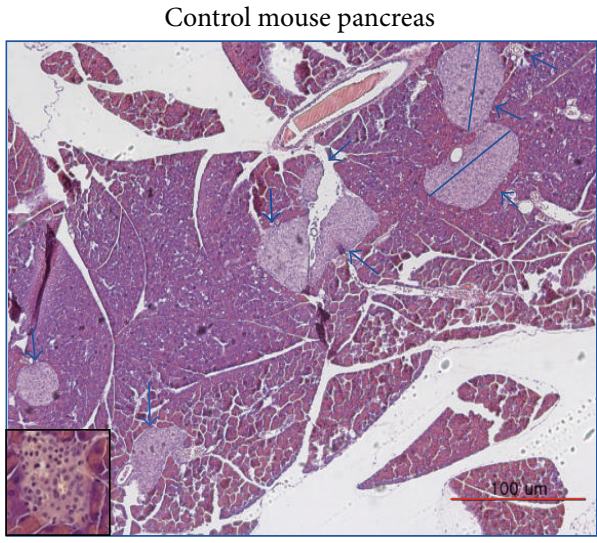

(a)

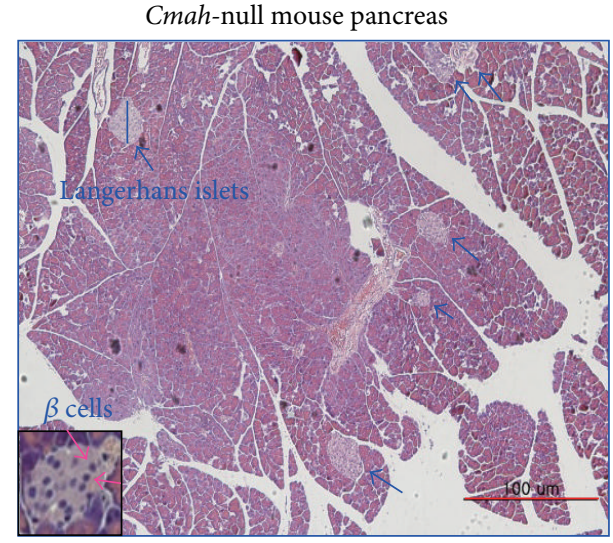

(b)

Figure 1: Representative pancreas tissues from control and Cmah-null mice. Islet size and number are significantly reduced in Cmah-null mouse compared to that of control mouse. Insert panels demonstrate that $\beta$-cell numbers in pancreas islets of Cmah-null mouse (b) were significantly lower than those of control (a).

2.6. Bioinformatics Analysis for Putative MicroRNA Targets and Pathway. miRNA target prediction and pathway analysis was performed using DIANA-mirPath. This allowed identification of molecular pathways potentially altered by the expression of single or multiple miRNAs (http://diana.imis.athena-innovation.gr/DianaTools/index .php? $r=$ mirpath/index) [21]. The software performs enrichment analysis of multiple miRNA target genes comparing each set of miRNA to all available pathways provided by the Kyoto Encyclopedia of Genes and Genomes (KEGG). Priority scores (enrichment $P$ value) are assigned based upon the predicted strength of the miRNA interactions with components of the target pathway.

2.7. Immunohistochemistry (IHC). For IHC, pancreatic tissues fixed in neutral buffer with $10 \%$ formalin were used. The tissues were embedded in paraffin and were sectioned at 3$5 \mu \mathrm{m}$. Sections used for the quantification of histopathology were stained with Hematoxylin QS to provide backgrounds. All photomicrographs were acquired using a Zeiss Axiophot microscope (Carl Zeiss, Oberkochen, Germany) equipped with an Olympus DP70 high-resolution digital microscope camera (Olympus, Center Valley, PA). The Sniper and Hematoxylin QS were all purchased from Vector Laboratories (Burlingame, CA).

2.8. Transfection with miRNA Mimic. Hsa-miR-15a-5p mimic, hsa-miR-29a-3p mimic, hsa-miR-29b-3p mimic, hsa-miR-1 mimic, and AllStar negative control siRNA were purchased from Qiagen (Valencia, CA, USA). HepG2 (liver hepatocellular carcinoma) cell line was incubated in Dulbecco's modified Eagle's medium (DMEM, Invitrogen, Carlsbad, CA, USA) with 10\% FBS. Cells were washed with PBS and the transfection was performed by using HiPerFect Transfection Reagents according to manufacturer's protocol (Qiagen, Valencia, CA). The final concentrations of the transfectants (hsa-miR-15a-5p mimic, hsa-miR-29a-3p mimic, and hsa-miR-29b-3p mimic) and their respective controls (AllStar siRNA for negative control and hsa-miR-1 mimic for positive control) were either $10 \mathrm{nM}$ (miRNA mimic) or $50 \mathrm{nM}$ (siRNAs). After $4 \mathrm{~h}$, transfection medium was replaced by fresh cell culture medium and cells were incubated for another $48 \mathrm{~h}$. For further analyses cells were then harvested by trypsin/EDTA.

\section{Results}

3.1. Altered miRNA Expression in the Liver and Pancreas of Cmah-Null Mice. To minimize differences in age, genetic background, sex, and time of sacrifice, which could affect metabolism, all animals with same age, sex, and genetic background were sacrificed at Zeitgeber time 9 (3 hrs before lights off). When Cmah homozygous mice with same age and sex from $\mathrm{Cmah}^{+/} \times \mathrm{Cmah}^{+/-}$crosses were examined and weighted, homozygous Cmah-null mice were indistinguishable from their wild type or heterozygous littermates in all respects. However, in both size and numbers of pancreatic islets in Cmah-null mice were significantly decreased (Figure 1). Also numbers of $\beta$-cells in pancreatic islets were significantly lower than those of control (insert panels of Figure 1). Consistent with a previous report $[9,11]$, however, germline inactivation of the Cmah gene has only a minor effect on development such as hearing loss in old age, skin healing delay in adulthood, and abnormal B-cell numbers. Taken together, these observations suggest that Cmah-null mice are closely associated with dysfunction of pancreatic islets or B-cells.

To examine differential expression of miRNAs between Cmah-null and control mouse livers, Liver miFinder miRNA PCR array analysis was performed $(n=6)$. Following array processing and normalization of raw array data, eight miRNAs were detected as differentially expressed. This included 


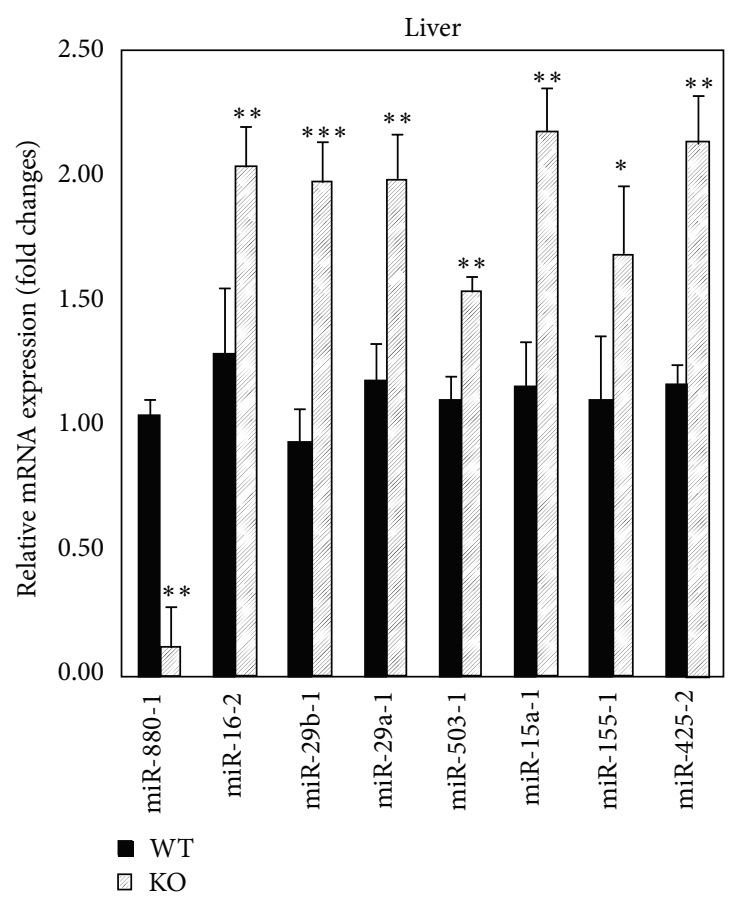

(a)

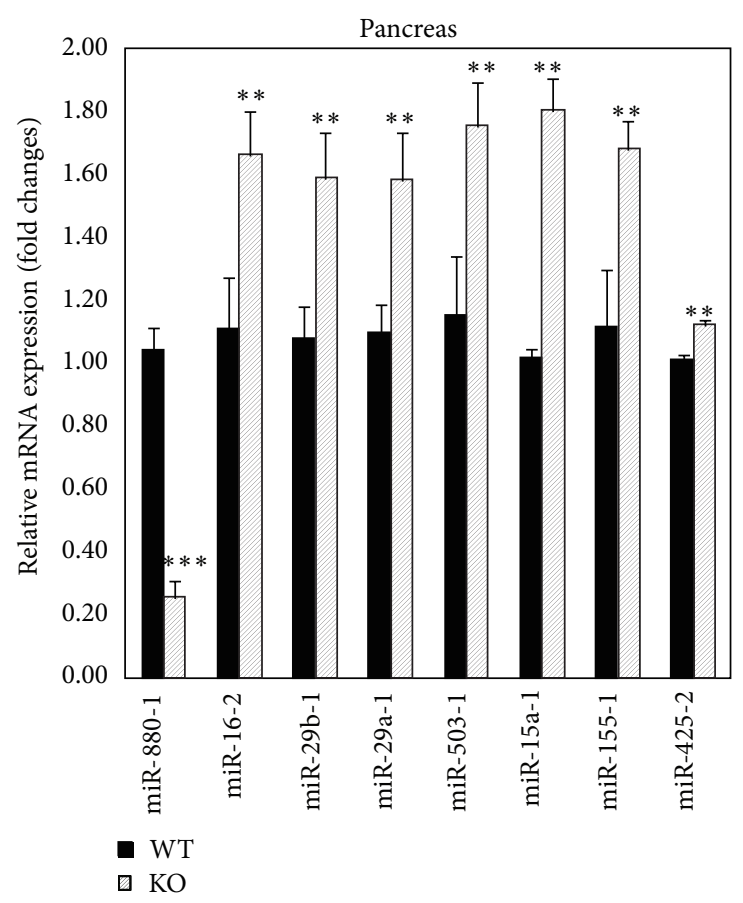

(b)

FIGURE 2: Validation of PCR array data by RT-qPCR. RT-qPCR analysis was performed using differentially expressed miRNAs in liver (a) and pancreas (b) RNA samples previously profiled by PCR array. Triplicate assays were carried out for each RNA sample and the relative amount of each miRNA was normalized to U6 snRNA. Data are expressed as fold changes of miRNA in livers and pancreas of Cmah-null versus control (C57BL/6) mice (mean $\pm \mathrm{SE}, n=3)\left({ }^{*} \mathrm{P}<0.05 ;{ }^{* *} \mathrm{P}<0.01 ;{ }^{* * *} \mathrm{P}<0.001\right)$.

seven (87.5\%; miR-16-5p, miR-29b-3p, miR-29a-3p, miR503-5p, miR-15a-5p, miR-155-5p, and miR-425-5p) that were significantly upregulated and one (12.5\%; miR-880-3p) that was downregulated ( $>2$ folds, $P<0.05$ ). To validate these findings, differentially expressed miRNAs identified by array were analyzed by RT-qPCR. The RT-qPCR data was consistent with the results of the array. This suggests that the data set obtained from array analysis accurately reflects the differential miRNA expression between the Cmah-null and control mice (Figure 2).

\subsection{Identification of Putative Target Pathways Using In Sil-} ico Analysis. To identify the biological pathways overrepresented among the predicted targets of differentially expressed miRNAs in Cmah-null derived livers and pancreas, we examined pathway enrichment analysis by using DIANAmirPath. The result showed putative pathways to be involved in a broad range of biological processes associated with the target genes (Table 1). Further, an investigation was carried out in statistically significant signaling pathways regulated by selected miRNAs. Among them, we found two major signal pathways such as insulin signaling (miR-155-5p, miR-425-5p, and miR-15a-5p) and PI3K-AKT signaling (miR-503-5p, miR16-5p, miR-29a-3p, and miR-29b-3p) pathways (Table 2). The percentage of genes involved in the insulin/PI3K-AKT signaling pathway was higher than for other pathways. The biological processes in which these genes (Pik3r1, Prkar2a, Sos1, Pik3r3, Fasn, Exoc7, Mapk3, Flot2, Raf1, and mTOR) are involved are shown in Table 2.
3.3. Cmah-Null Mice Have Defects in the Insulin/PI3K-AKT Signaling Pathway. To determine whether the loss of Cmah was responsible for differential expression of miRNAs, RTqPCR and western blot analysis were performed using the liver and pancreas of Cmah-null and control mice. Cmahnull livers had significant downregulation of IRS2, AKT, and Foxol mRNA expression, whilst Foxol mRNA expression was significantly upregulated in the pancreas (Figure 3(a)). PI3Ks are composed of a catalytic subunit (p110) and a regulatory subunit. Various isoforms of the catalytic subunit (p110 $\alpha, \mathrm{p} 110 \beta, \mathrm{p} 110 \gamma$, and $\mathrm{p} 110 \delta$ ) have been isolated, and the regulatory subunits that associate with $\mathrm{p} 110 \alpha, \mathrm{p} 110 \beta$, and $\mathrm{p} 110 \delta$ are $\mathrm{p} 85 \alpha$ and $\mathrm{p} 85 \beta$. Among them, we used the $\mathrm{p} 110 \alpha$ isoform of PI3K, because it plays a fundamental role in insulin signaling and control of hepatic glucose and lipid metabolism [22]. In addition, Akt is activated by phospholipid binding and activation loop phosphorylation at Thr308 by PDK1 and by phosphorylation within the carboxy terminus at Ser473. Therefore, we used the p-AKT (Phospho-Akt [Thr308]) antibody in this study.

RT-qPCR and western blotting analysis confirmed that mRNA and protein levels of IRS2, PI3K, and p-AKT were reduced in livers from Cmah-null mice (Figures 3(a) and 3(b)). This suggests that dysregulation of miRNA may affect both mRNA stability and protein translation. To confirm this possibility, we analyzed mRNA expression of genes involved in insulin/PI3K-AKT signaling by RT-qPCR. Expression of three randomly selected genes was significantly lower in Cmah-null mice than in controls (Figure 4(a)). As shown 


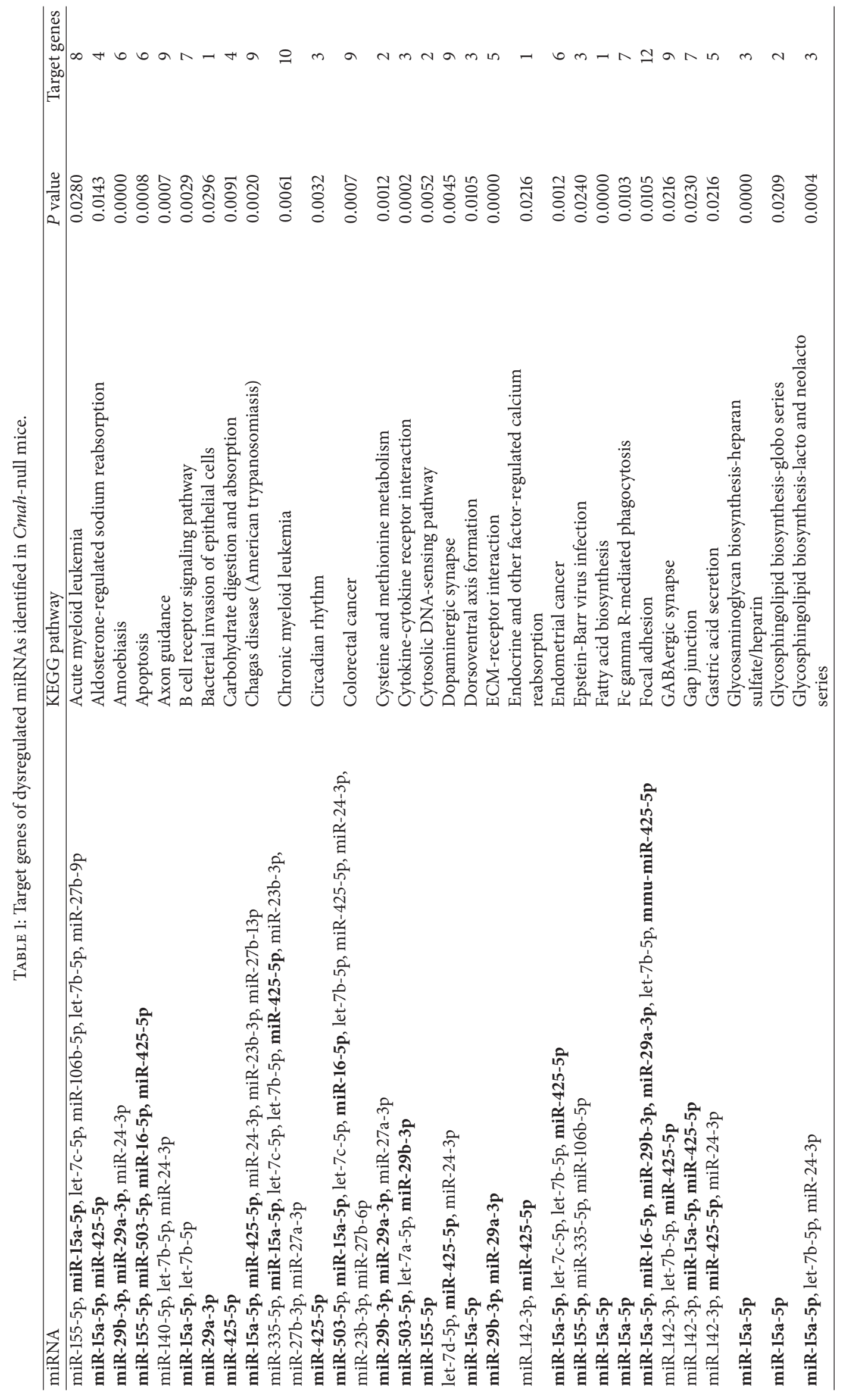




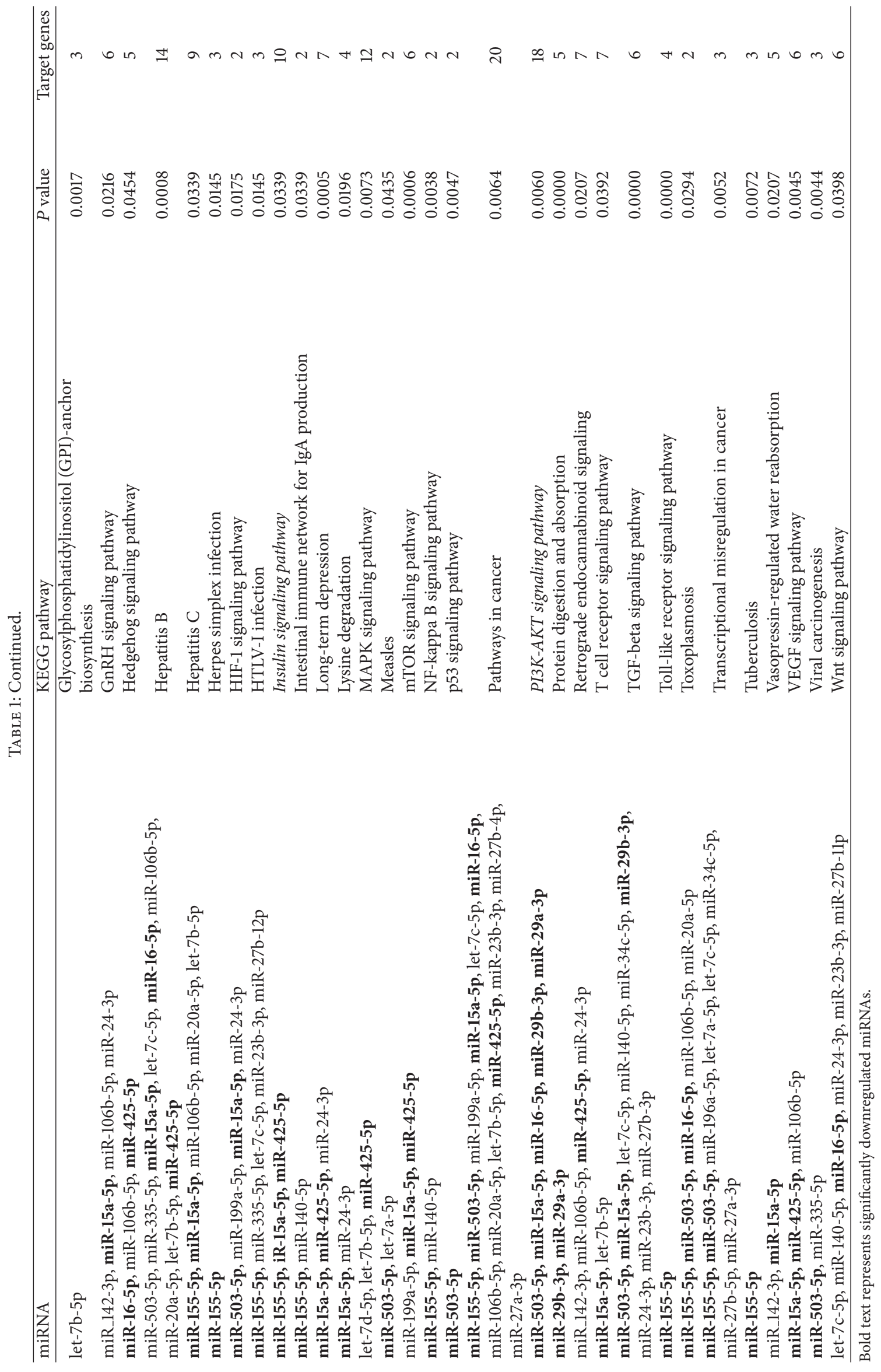


TABLE 2: KEGG pathway analysis of miRNA target genes.

\begin{tabular}{lccl}
\hline miRNAs & $\begin{array}{c}\text { Significant } \\
\text { pathway }\end{array}$ & P value & Target genes \\
\hline $\begin{array}{l}\text { miR-155-5p, } \\
\text { miR-15a-5p, } \\
\text { miR-425-5p }\end{array}$ & $\begin{array}{c}\text { Insulin } \\
\text { signaling } \\
\text { pathway }\end{array}$ & 0.0339 & $\begin{array}{l}\text { Rheb, Socs1, Pik3r1, Eif4e2, Prkar2a, Prkx, Crkl, Sos1, } \\
\text { Bad, Acaca, Pik3r3, Fasn, Exoc7, Mapk3, Flot2, Raf1, } \\
\text { miR-503-5p, }\end{array}$ \\
$\begin{array}{l}\text { miR-15a-5p, } \\
\text { miR-16-5p, } \\
\text { miR-29b-3p, }\end{array}$ & & \\
miR-29a-3p & PI3K-Akt & \\
\hline
\end{tabular}
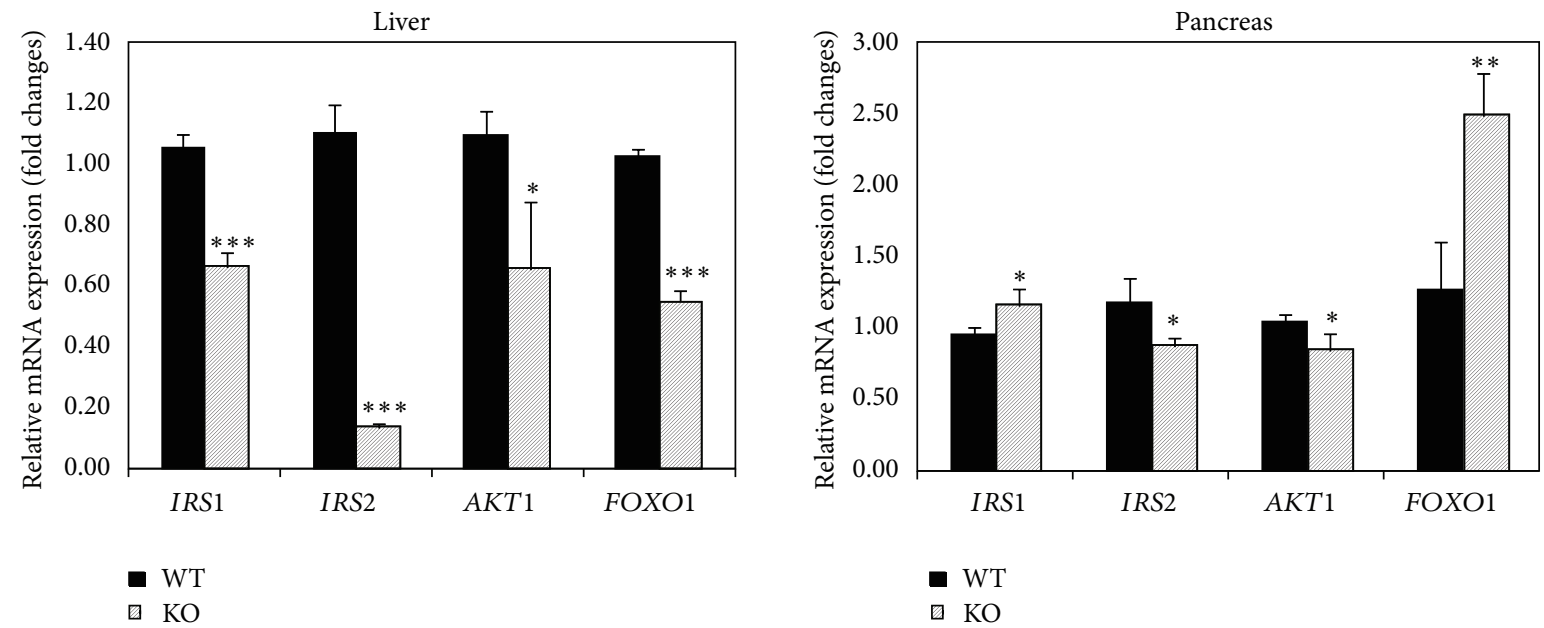

(a)
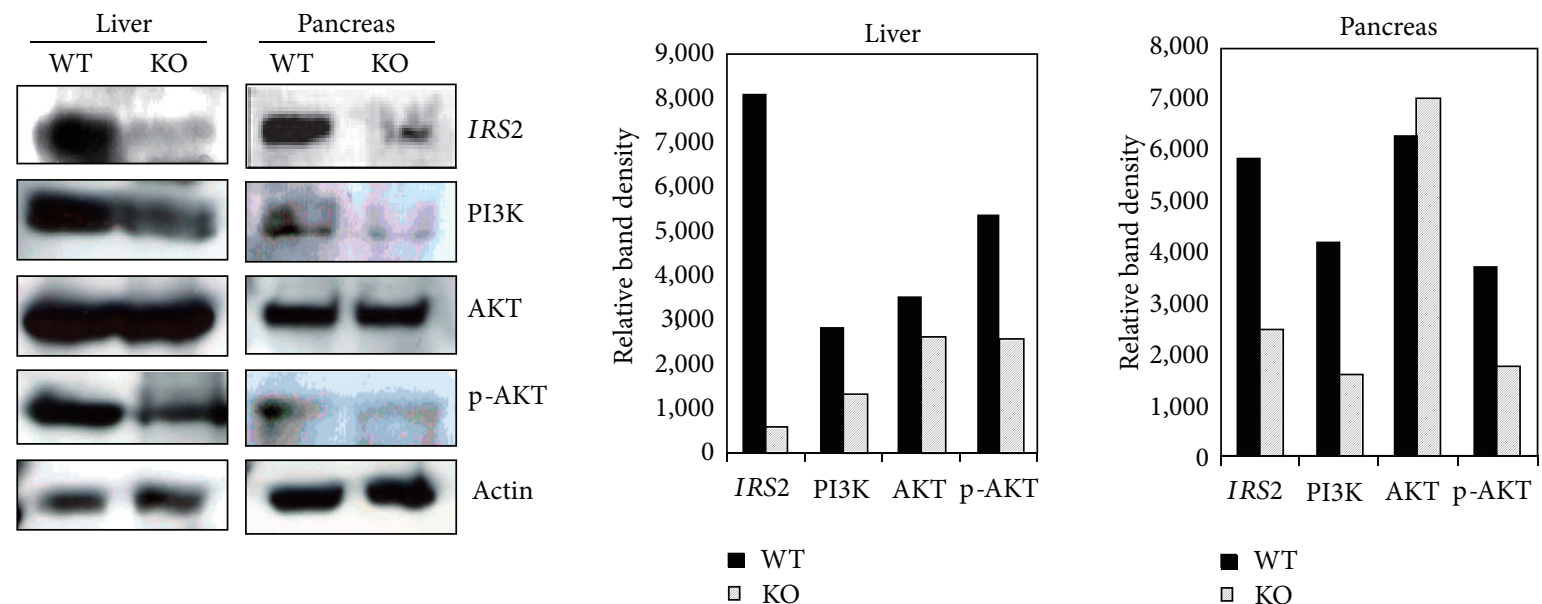

(b)

FIGURE 3: Expression changes in the insulin/PI3K-AKT signaling pathway. (a) Expression levels of IRS1, IRS2, AKT1, and FOXO1 mRNAs in livers (left) and pancreas (right) of control and Cmah-null mice were determined using real-time reverse transcription PCR (RT-qPCR) $\left({ }^{*} P<0.05 ;{ }^{* *} P<0.01 ;{ }^{* * *} P<0.001\right)$. (b) Western blot analysis of proteins involved in the target pathway in liver (left) and pancreas (right) of control and Cmah-null mice. Actin was used as a loading control. Band intensities were quantified by image processing and analysis was made using ImageJ v1.32. 

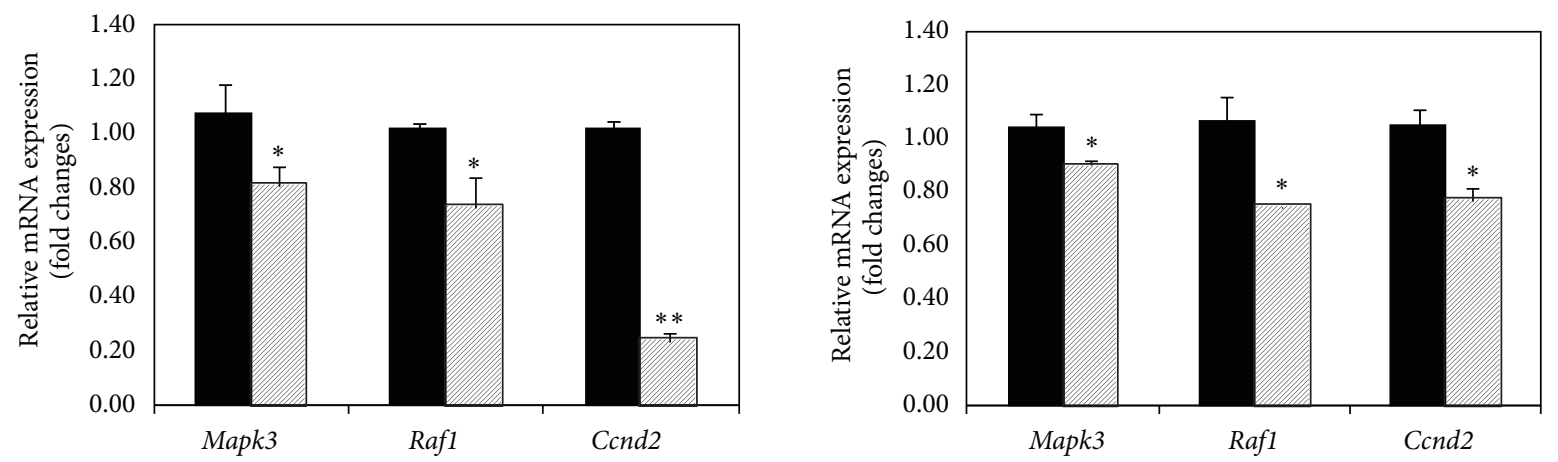

- WT

- $\mathrm{WT}$

圆 $\mathrm{KO}$

圆O

(a)

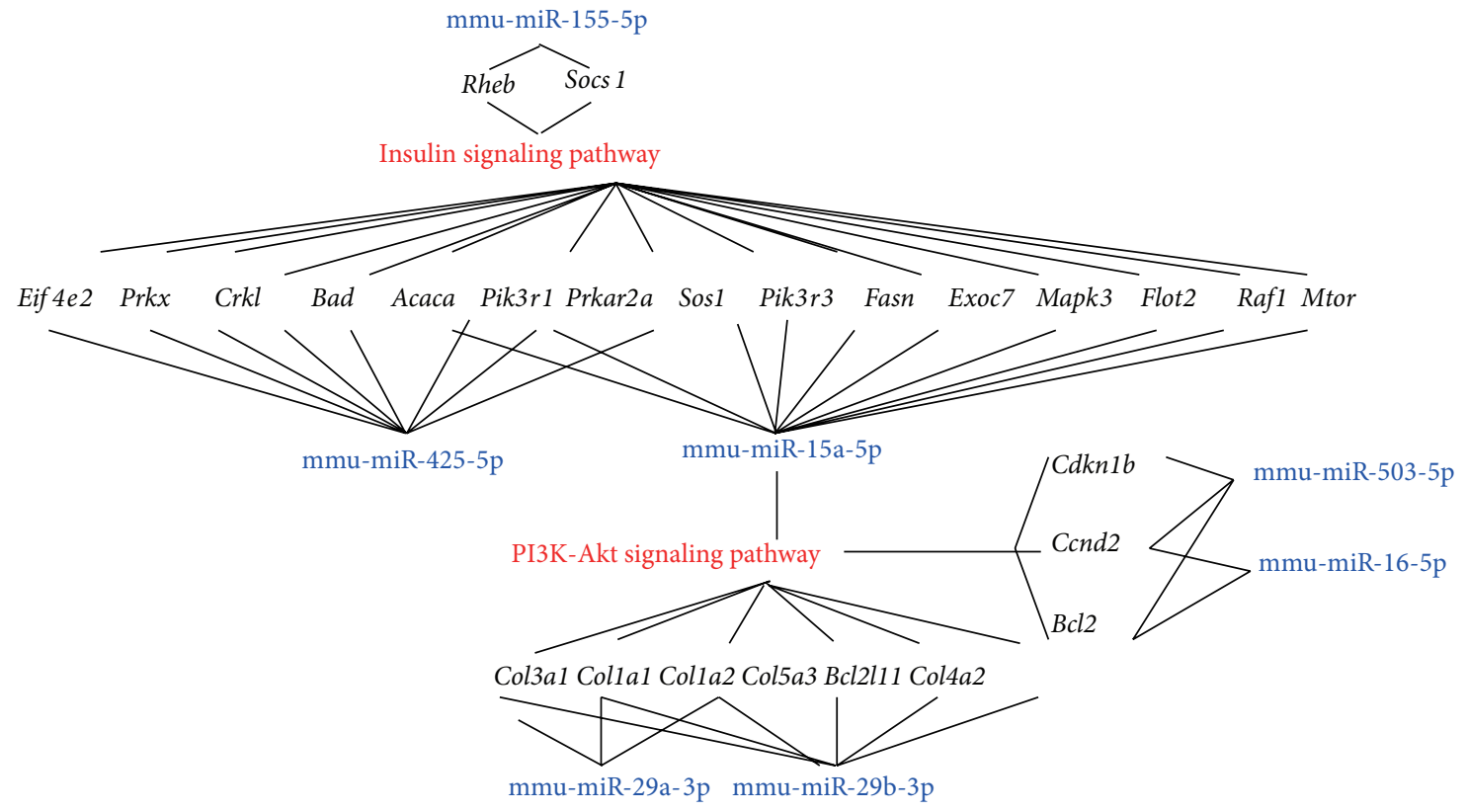

(b)

FIGURE 4: Identification of target genes of dysregulated miRNAs in Cmah-null mice. (a) RT-qPCR analysis was performed using randomly selected genes (Mapk3, Rafl, and Ccnd2), which involved the insulin/PI3K-AKT signaling pathway. Expression of mRNAs in livers (left) and pancreas (right) of control and Cmah-null mice were downregulated $\left({ }^{*} P<0.05\right.$; $\left.{ }^{* *} P<0.01\right)$. (b) Dysregulated miRNAs and their target genes which involved in insulin/PI3K-AKT signaling pathway in liver and pancreas of Cmah-null mouse. Red and blue present the refined pathways and putative miRNAs, respectively. Black indicates putative target genes for their pathway.

in Figure 4(b), we proposed an interaction of miRNAs with key components of the insulin/PI3K-AKT signaling pathway. This putative insulin/PI3K-AKT signaling pathway suggested that functional loss of Cmah gene during human evolution is closely associated with Type II diabetes due to altered miRNAs and target genes interaction.

3.4. Expression Validation of Target Genes by Using Three Different miRNAs. To determine whether the miRNAs dysregulate expression of their predicted target genes, we transfected 3 different types of miRNA mimics (hsa-miR-15a-5p mimic, hsa-miR-29a-3p mimic, and hsa-miR-29b-3p mimic) and 1 positive (hsa-miR-1 mimic) or negative control siRNA
(AllStar negative control siRNA) into HepG2 cells. At 48 hrs after transfection, each cell was harvested and was provided for RT-qPCR analysis. In this study, the hsa-miR-1 mimic and AllStar siRNA were used as positive control and negative control for miRNA mimic experiments, respectively. A positive control, has-miR-1 mimic, markedly decreased the expression of HDAC4 in HepG2, compared to negative control group, whereas a negative control do not altered expression of HDAC4 gene (Figure 5(a)). We next determined the effect of miR-15a on mRNA expression of Fasn, Colla2, Col4a2, and Ccnd2 genes. Transfection of HepG2 cells with miR15a mimic significantly decreased the mRNA level of Colla2, Col4a2, and Ccnd2, whereas Fasn mRNA expression was not changed 


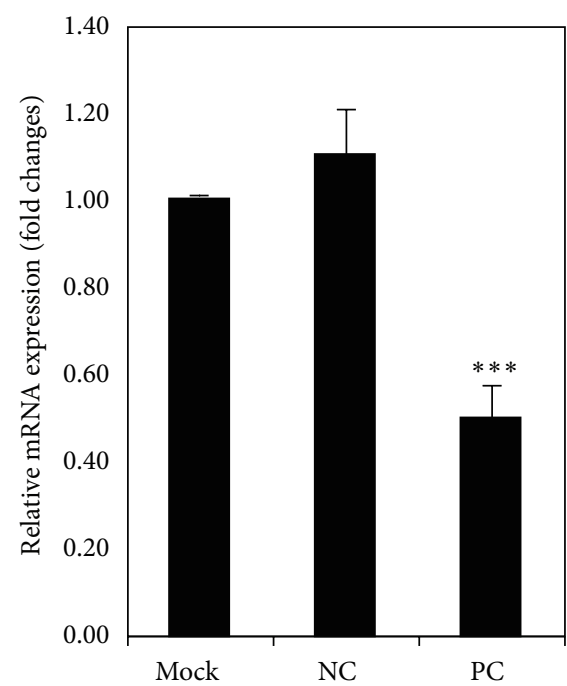

HDAC4

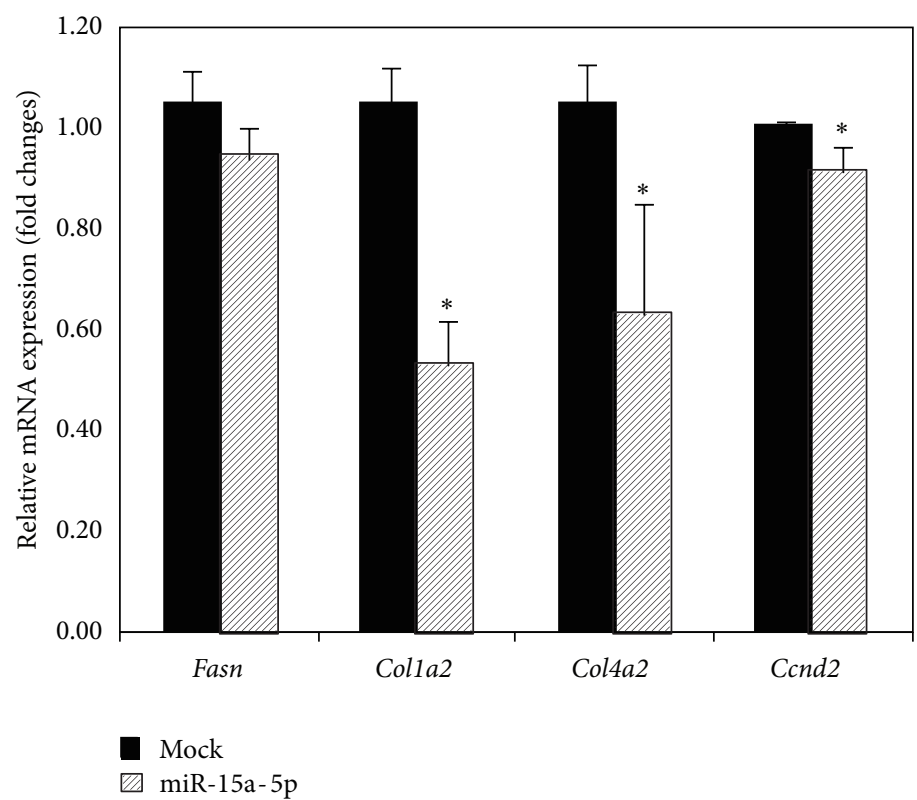

(b)

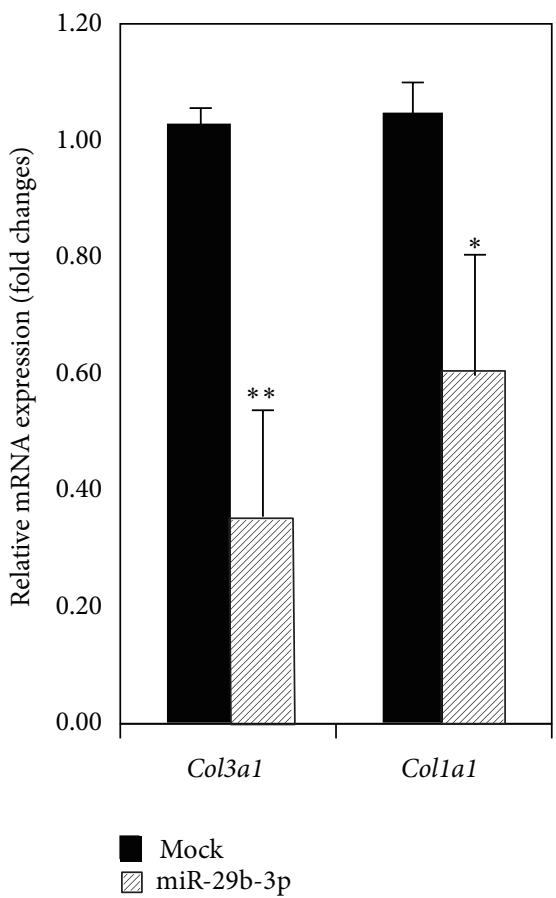

(d)

FIGURE 5: Posttranscriptional regulation of identified target genes by dysregulated miRNAs (miR-15a or miR-28a and b). (a) HDAC4 transcript level as a positive or negative control was analyzed by RT-qPCR after transfection using $10 \mathrm{nM}$ miR-1 mimic or AllStar negative control siRNA. Mock: no transfection of miRNA mimic, NC: negative control (transfection of $50 \mathrm{nM}$ AllStar negative control siRNA), PC: positive control (transfection of $10 \mathrm{nM}$ miR-1 mimic). (b) RT-qPCR analysis of miR-15a predicted target genes. The transfection of miR-15a-5p mimic into HepG2 cells downregulates putative target gene mRNAs expression. (c) and (d) RT-qPCR analysis of miR-29a or -29b predicted target genes. The transfections of miR-29a-3p mimic or miR-29b-3p mimic into HepG2 cells downregulate their putative target gene mRNAs expression, respectively. The experiments were independently repeated three times and GAPDH served as internal control. $\left({ }^{*} P<0.05\right.$; ${ }^{* *} P<0.01$; $\left.{ }^{* * *} P<0.001\right)$. 
(Figure 5(b)). Furthermore, HepG2 cells transfected with miR-29a and miR-29b mimic significantly downregulated its target mRNA expression such as Col3al and Collal genes, compared to those of mock group (Figures 5(c) and 5(d)). Taken together, our observation strongly demonstrated that miRNAs dysregulation in Cmah-null mouse-derived liver could impair insulin/PI3K-AKT expression signaling during transition from glucose tolerance to intolerance.

\section{Discussion}

This study observed that expression of the miRNAs miR-1555p, miR-425-5p, miR-15a-5p, miR-503-5p, miR-16-5p, miR$29 a-3 p$, and miR-29b-3p in the liver of Cmah-null mice may downregulate components of the insulin/PI3K-AKT signaling pathway in concert with other genes. These include Rheb, Socs1, Eif4e2, Prkx, Crkl, Bad, Acaca, Pik3r1, Prkar2a, Sos1, Pik3r3, Fasn, Exoc7, Mapk3, Flot2, Raf1, Mtor, Col3a1, Colla1, Col1a2, Col5a3, Bcl2l11, Col4a2, Cdkn1b, Ccnd2, and Bcl2.

miRNAs play a key role in regulating many physiological and pathological processes in humans, including diabetes and the related metabolic syndrome. It has been well demonstrated that miRNAs such as miR-375, miR-29, miR-320, miR103 , miR-107, and miR-126, play a crucial role in regulating glucose and lipid metabolism through control of pancreatic islet cell function, adipocyte insulin resistance, hepatocyte insulin signaling, and glucose homeostasis [15-19]. In this study, differential miRNA expression was found in Cmahnull mice liver using Liver miFinder miRNA PCR array. The majority of miRNAs differentially affected included genes encoding components of the insulin/PI3K-AKT signaling pathway. As the liver consumes more oxygen at rest than other organs and is thought to play a role in the systemic control of glucose and lipid utilization [23], gene expression and metabolic measurements in the liver were studied. These metabolic pathways are partly regulated at the transcriptional level, perturbation of which leads to pathological changes. In livers of Cmah-null mice, a rapid increase in miRNA expression was observed. Consistent with these results, insulin/PI3K-AKT signaling-related gene expression was significantly decreased. These results suggest that the key molecules altered in the pathway in Cmah-null mice are associated with a specific group of miRNAs.

Heterogeneous genetic background and lifestyle make it difficult to study the mechanism(s) of metabolic syndrome in humans. Presently, mouse models are a popular tool used to study human metabolic disorder as they provide evidence that can be extrapolated to validate the human condition. Because the phenotype of HFD-fed Cmah-null mice is similar to that of patients with type 2 diabetes, the Cmah-null mouse is one of the most suitable animal models of metabolic syndrome. It is not known, however, how impaired glucose tolerance and $\beta$-cell dysfunction arise in HFD-fed Cmah-null mice. To fill the gap of the phenotype of mice in relation to the previous observations [12], differential miRNA expression was examined in livers from control and Cmah-null mice using a pathway-focused glucose metabolism PCR array (data not shown). In Kyoto Encyclopedia of Genes and Genomes (KEGG), the molecular pathway analysis identified miRNAs in the upregulated or downregulated genes. Each case was linked to at least two different cellular pathways even if crosslinking was observed at low levels. Network-1 shows insulinrelated signaling pathway (Table 2 and Figure 4(b) and includes 17 genes: Rheb, Socs1, Pik3r1, Eif4e2, Prkar2a, Prkx, Crkl, Sos1, Bad, Acaca, Pik3r3, Fasn, Exoc7, Mapk3, Flot2, Raf1, and Mtor. Network-2 functions in PI3K-AKT signaling pathway (Table 2 and Figure 4(b) and includes 19 genes: Pik3r1, Prkar2a, Sos1, Pik3r3, Fasn, Exoc7, Mapk3, Flot2, Raf1, Mtor, Col3a1, Col1a1, Col1a2, Col5a3, Bcl2l11, COl4a2, $C d k n 1 b, C c n d 2$, and $B c l 2$. These data suggest that simultaneous impairment of insulin processes required for normal liver function could contribute to the development of metabolic disorders such as hyperglycemia and diabetes in Cmah-null mice.

Pirola et al. [24] suggested that liver cells mimic the effects of hyperinsulinemia, leading to downregulation of both PI3K/AKT signaling pathways and glucose uptake via a decrease in IRS-1/2 docking molecules. Because PI3K is the key molecule controlling the decrease in IRS-1/2 as downregulation of IRS-1/2 is effectively prevented by PI3K inhibition [24]. The same mechanism could be involved in liver cells of Cmah-null mouse. Definitely, for IRS-1/2, a PI3Kdependent pathway, with PI3K itself acting as an IRS-1 serine/threonine kinase, appears to be involved, although IRS-2 degradation is controlled by a PI3K-mTOR-dependent mechanism. Moreover, tissue-specific mechanisms may control IRS degradation, as IRS-1 degradation in adipocytes appears to be mTOR-dependent $[24,25]$. The degradation of IRS proteins is promoted by serine/threonine phosphorylation and provides a molecular link to insulin resistance $[26,27]$.

In this study, mRNA and protein levels of IRS2 in the livers of Cmah-null mice were significantly downregulated. As a result, IRS1 and IRS2 downstream, AKT1 and $m$ TOR mRNA expression is also downregulated. As shown in Figure 4(b) miR-155-5p miR-15a-5p, and miR-425-5p in the case of insulin signaling and miR-29b-3p, miR-29a-3p, miR-16-5p, and miR-503-5p in the case of PI3K-AKT1-mTOR signaling were significantly dysregulated. This suggests that an insulindependent pathway in Cmah-null mice causes the increased glucose production. Previous studies showed that mice bearing a human-specific Cmah genetic mutation developed an obesity-related metabolism and diabetes [12]. It was shown that sialylation with Neu5Gc plays an important role in insulin production by $\beta$ cells and that its loss contributes to $\beta$-cell dysfunction in mice. Taken together, these studies highlight the potential of the Cmah-null mouse to enhance scientific understanding of human metabolic syndrome.

\section{Conclusion}

Differential miRNA expression was found in Cmah-null mice liver using Liver miFinder miRNA PCR array. Among them, miR-155-5p, miR-425-5P, miR-15a-5p, miR-503-5p, miR-16$5 p$, miR-29a-3p, and miR-29b-3p were significantly upregulated in the liver and pancreas of Cmah-null mice. These target miRNAs are closely associated with dysregulation of insulin/PI3K-AKT signaling, suggesting that the Cmah-null mice could be a useful model for studying diabetes. 
Abbreviations

Cmah: CMP-Neu5Ac hydroxylase

HFD: High-fat diet

miRNAs: MicroRNAs

Neu5Ac: $\quad N$-acetylneuraminic acid

Neu5Gc: $\quad N$-glycolylneuraminic acid

KEGG: Kyoto Encyclopedia of Genes and Genomes

RT-qPCR: Real-time reverse transcription quantitative polymerase chain reaction.

\section{Conflict of Interests}

The authors declare that there is no conflict of interests regarding the publication of this paper.

\section{Acknowledgment}

This work was supported by next generation of Biogreen 21 (PJ009625) from the Rural Development Administration (RDA), Republic of Korea.

\section{References}

[1] H. Smit, W. Gaastra, J. P. Kamerling, J. F. Vliegenthart, and F. K. de Graaf, "Isolation and structural characterization of the equine erythrocyte receptor for enterotoxigenic Escherichia coli K99 fimbrial adhesin," Infection and Immunity, vol. 46, no. 2, pp. 578-584, 1984.

[2] R. Laufs and J. Heesemann, "Serologic diagnosis and prophylaxis of virus hepatitis," Offentliche Gesundheitswesen, vol. 46, no. 11, pp. 578-581, 1984.

[3] H.-H. Chou, H. Takematsu, S. Diaz et al., "A mutation in human CMP-sialic acid hydroxylase occurred after the Homopan divergence," Proceedings of the National Academy of Sciences of the United States of America, vol. 95, no. 20, pp. 11751-11756, 1998.

[4] S. Kelm, R. Schauer, and P. R. Crocker, "The Sialoadhesins-a family of sialic acid-dependent cellular recognition molecules within the immunoglobulin superfamily," Glycoconjugate Journal, vol. 13, no. 6, pp. 913-926, 1996.

[5] A. Irie, S. Koyamat, Y. Kozutsumi, T. Kawasaki, and A. Suzuki, "The molecular basis for the absence of N-glycolylneuraminic acid in humans," The Journal of Biological Chemistry, vol. 273, no. 25, pp. 15866-15871, 1998.

[6] T. Hayakawa, Y. Satta, P. Gagneux, A. Varki, and N. Takahata, "Alu-mediated inactivation of the human CMP-N-acetylneuraminic acid hydroxylase gene," Proceedings of the National Academy of Sciences of the United States of America, vol. 98, no. 20, pp. 11399-11404, 2001.

[7] A. Varki, "Uniquely human evolution of sialic acid genetics and biology," Proceedings of the National Academy of Sciences of the United States of America, vol. 107, no. 2, pp. 8939-8946, 2010.

[8] N. M. Varki, E. Strobert, E. J. Dick Jr., K. Benirschke, and A. Varki, "Biomedical differences between human and nonhuman hominids: potential roles for uniquely human aspects of sialic acid biology," Annual Review of Pathology: Mechanisms of Disease, vol. 6, pp. 365-393, 2011.

[9] M. Hedlund, P. Tangvoranuntakul, H. Takematsu et al., "Nglycolylneuraminic acid deficiency in mice: implications for human biology and evolution," Molecular and Cellular Biology, vol. 27, no. 12, pp. 4340-4346, 2007.

[10] K. Chandrasekharan, J. H. Yoon, Y. Xu et al., "A human-specific deletion in mouse Cmah increases disease severity in the $\mathrm{mdx}$ model of duchenne muscular dystrophy," Science Translational Medicine, vol. 2, no. 42, Article ID 42ra54, 2010.

[11] Y. Naito, H. Takematsu, S. Koyama et al., "Germinal center marker GL7 probes activation-dependent repression of $\mathrm{N}$ glycolylneuraminic acid, a sialic acid species involved in the negative modulation of B-cell activation," Molecular and Cellular Biology, vol. 27, no. 8, pp. 3008-3022, 2007.

[12] S. Kavaler, H. Morinaga, A. Jih et al., "Pancreatic $\beta$-cell failure in obese mice with human-like CMP-Neu5Ac hydroxylase deficiency," The FASEB Journal, vol. 25, no. 6, pp. 1887-1893, 2011.

[13] K. Chen and N. Rajewsky, "The evolution of gene regulation by transcription factors and microRNAs," Nature Reviews Genetics, vol. 8, no. 2, pp. 93-103, 2007.

[14] Y. Li and K. V. Kowdley, "MicroRNAs in common human diseases.," Genomics, proteomics \& bioinformatics, vol. 10, no. 5, pp. 246-253, 2012.

[15] M. N. Poy, L. Eliasson, J. Krutzfeldt et al., "A pancreatic isletspecific microRNA regulates insulin secretion," Nature, vol. 432, no. 7014, pp. 226-230, 2004.

[16] A. He, L. Zhu, N. Gupta, Y. Chang, and F. Fang, "Overexpression of micro ribonucleic acid 29, highly up-regulated in diabetic rats, leads to insulin resistance in 3T3-L1 adipocytes," Molecular Endocrinology, vol. 21, no. 11, pp. 2785-2794, 2007.

[17] M. Trajkovski, J. Hausser, J. Soutschek et al., "MicroRNAs 103 and 107 regulate insulin sensitivity," Nature, vol. 474, no. 7353, pp. 649-653, 2011.

[18] M. N. Poy, J. Hausser, M. Trajkovski et al., "miR-375 maintains normal pancreatic $\alpha$ - and $\beta$-cell mass," Proceedings of the National Academy of Sciences of the United States of America, vol. 106, no. 14, pp. 5813-5818, 2009.

[19] H. Y. Ling, H. S. Ou, S. D. Feng et al., "Changes in microRNA (miR) profile and effects of miR-320 in insulin-resistant 3t311 adipocytes," Clinical and Experimental Pharmacology and Physiology, vol. 36, no. 9, pp. e32-e39, 2009.

[20] A. Dávalos, L. Goedeke, P. Smibert et al., "miR-33a/b contribute to the regulation of fatty acid metabolism and insulin signaling," Proceedings of the National Academy of Sciences of the United States of America, vol. 108, no. 22, pp. 9232-9237, 2011.

[21] G. L. Papadopoulos, P. Alexiou, M. Maragkakis, M. Reczko, and A. G. Hatzigeorgiou, "DIANA-mirPath: integrating human and mouse microRNAs in pathways," Bioinformatics, vol. 25, no. 15, pp. 1991-1993, 2009.

[22] V. R. Sopasakis, P. Liu, R. Suzuki et al., "Specific roles of the p110alpha isoform of phosphatidylinsositol 3-kinase in hepatic insulin signaling and metabolic regulation," Cell Metabolism, vol. 11, no. 3, pp. 220-230, 2010.

[23] W. Langhans, "Role of the liver in the control of glucoselipid utilization and body weight," Current Opinion in Clinical Nutrition and Metabolic Care, vol. 6, no. 4, pp. 449-455, 2003.

[24] L. Pirola, S. Bonnafous, A. M. Johnston, C. Chaussade, F. Portis, and E. van Obberghen, "Phosphoinositide 3-kinase-mediated reduction of insulin receptor substrate-1/2 protein expression via different mechanisms contributes to the insulin-induced desensitization of its signaling pathways in L6 muscle cells," The Journal of Biological Chemistry, vol. 278, no. 18, pp. 15641-15651, 2003. 
[25] T. Haruta, T. Uno, J. Kawahara et al., "A rapamycin-sensitive pathway down-regulates insulin signaling via phosphorylation and proteasomal degradation of insulin receptor substrate-1," Molecular Endocrinology, vol. 14, no. 6, pp. 783-794, 2000.

[26] T. M. Pederson, D. L. Kramer, and C. M. Rondinone, "Serine/ threonine phosphorylation of IRS-1 triggers its degradation: possible regulation by tyrosine phosphorylation," Diabetes, vol. 50, no. 1, pp. 24-31, 2001.

[27] Y. Zick, "Insulin resistance: a phosphorylation-based uncoupling of insulin signaling," Trends in Cell Biology, vol. 11, no. 11, pp. 437-441, 2001. 

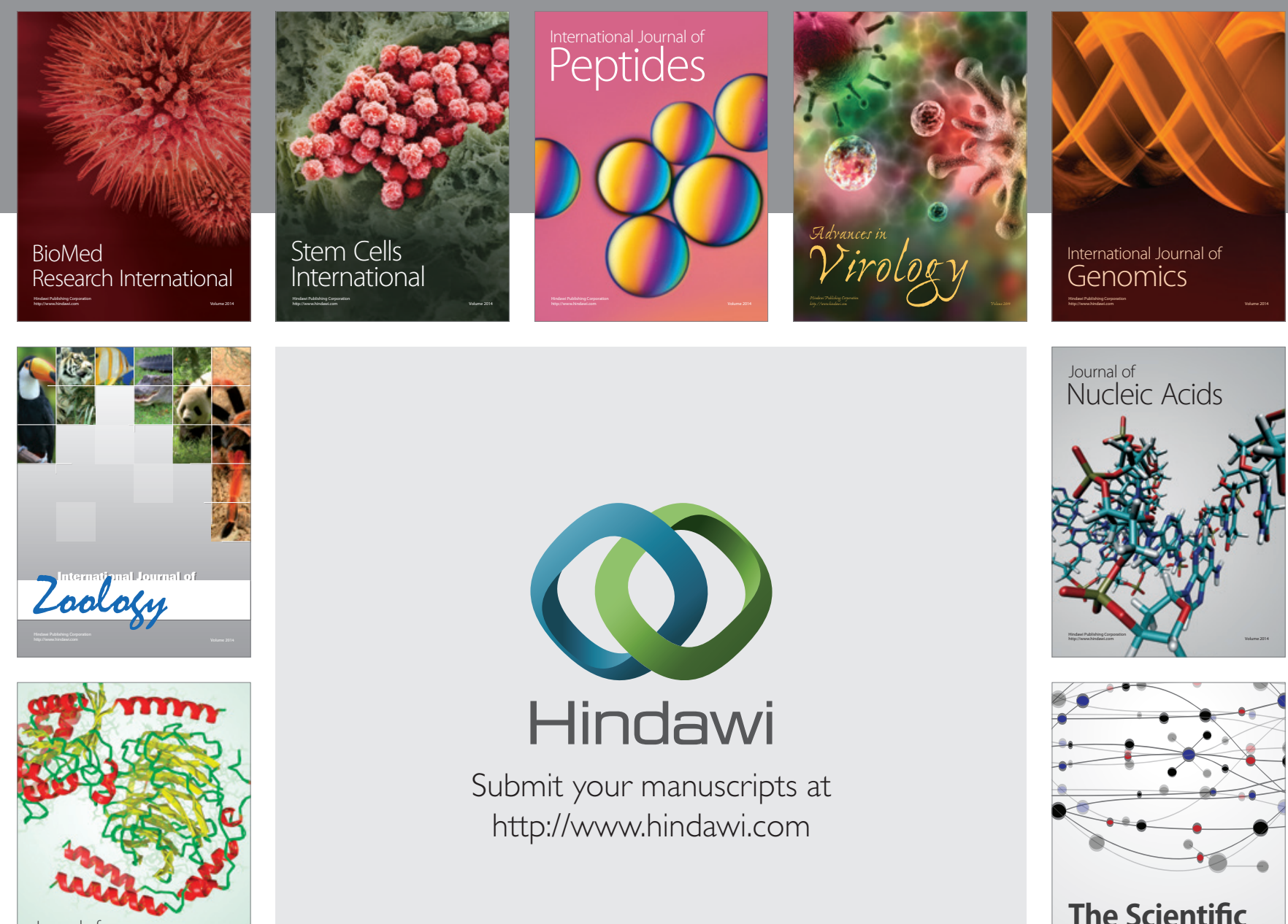

Submit your manuscripts at

http://www.hindawi.com

Journal of
Signal Transduction
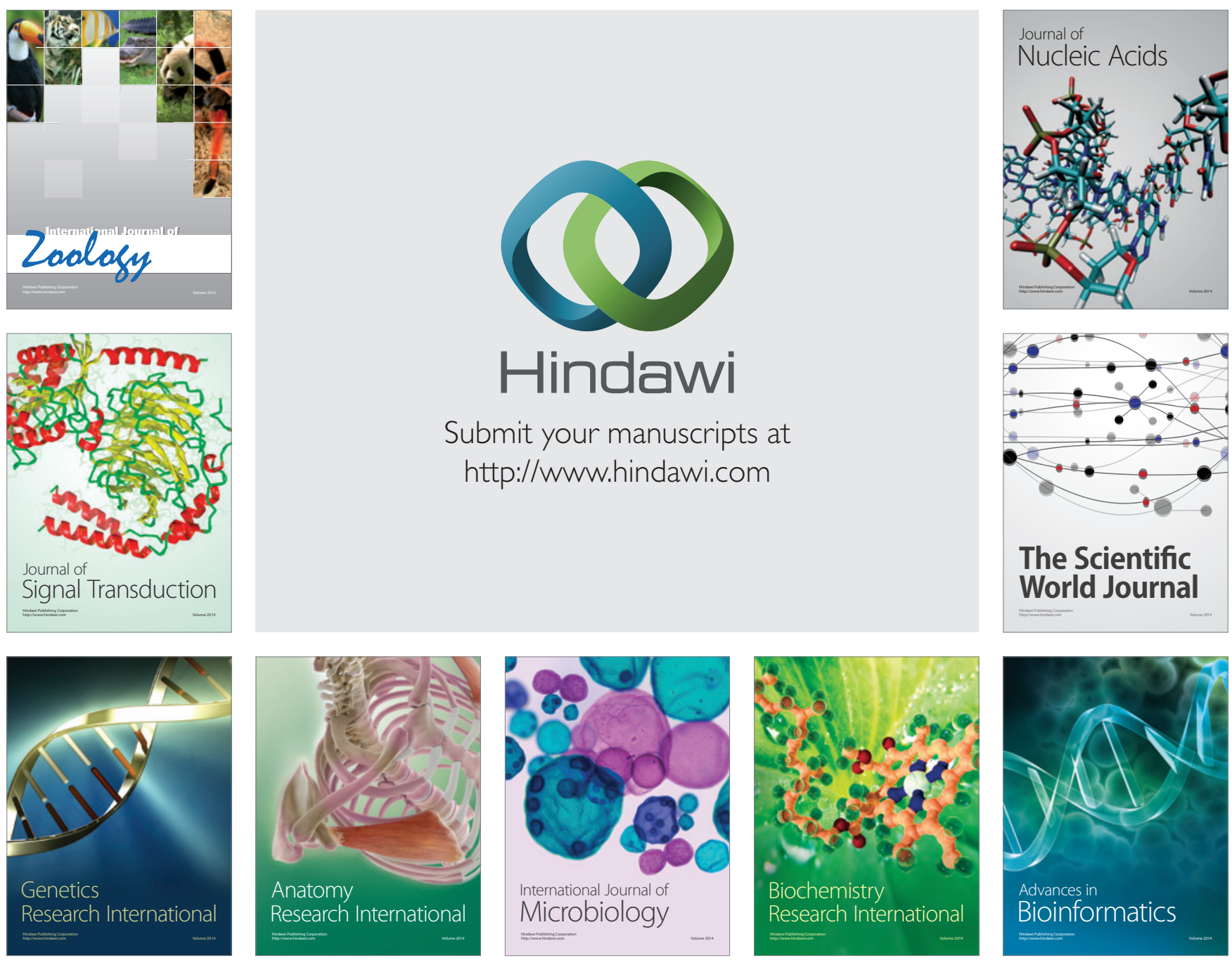

The Scientific World Journal
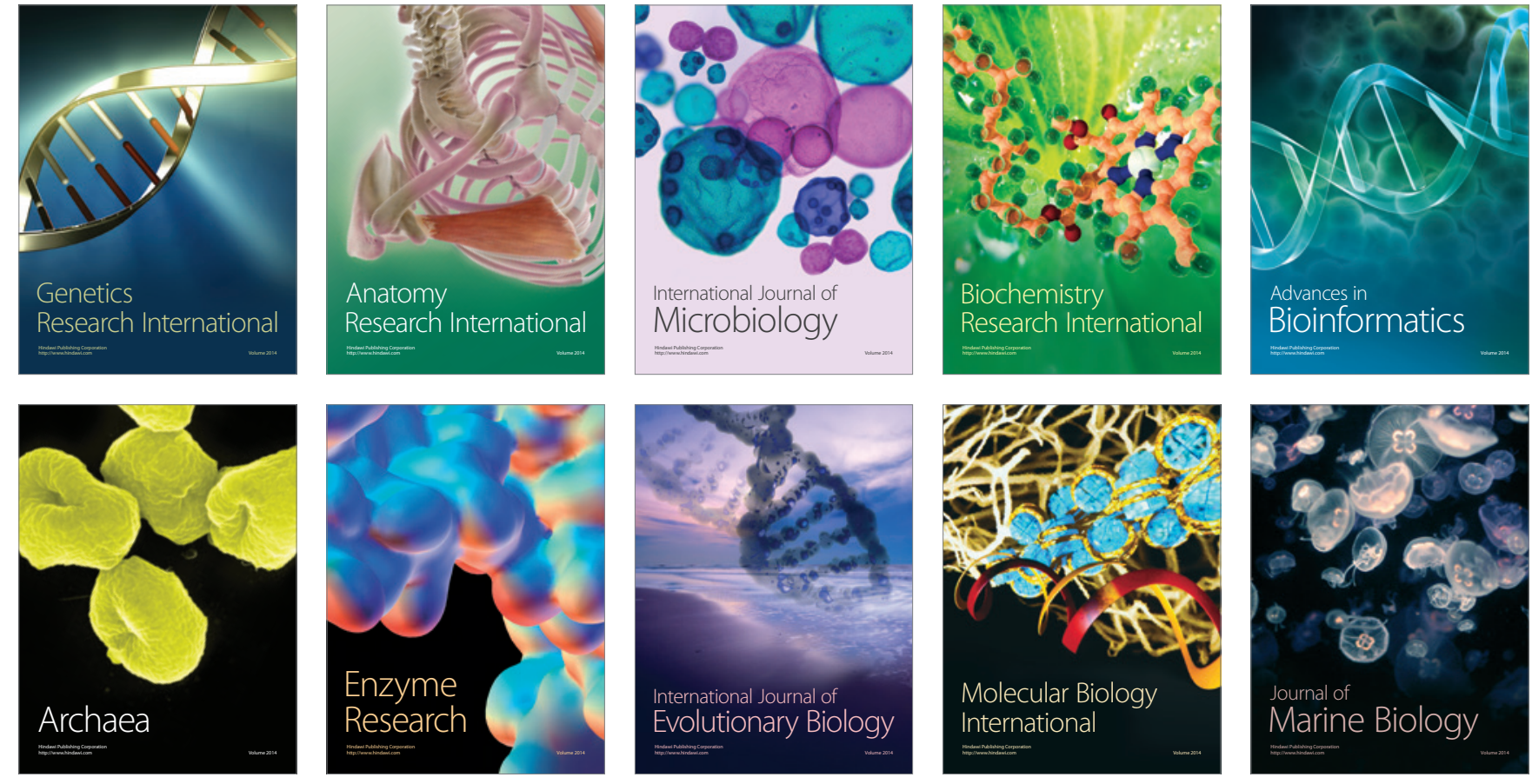